\title{
AUTONOMOUS COOPERATIVE UNMANNED AERIAL VEHICLE
}

\author{
A. N. Chaves ${ }^{1}$, P. S. Cugnasca ${ }^{1}$ \\ ${ }^{1}$ Department of Computer and Digital Systems Engineering (PCS), School of Engineering at \\ the University of São Paulo (aquila.chaves@gmail.com, paulo.cugnasca@poli.usp.br)
}

\begin{abstract}
As a quite interesting subject, there are an increasing number of researches about UAV (Unmanned Aerial Vehicle) in the literature. These robots are quite suitable to dull, dirty and dangerous missions (e.g., over flights at low altitude, especially at night, and longrunning operations, which exposes the crew to extreme fatigue). Thus, an important application of these vehicles is the search operations involving multiple UAVs. However, despite the huge potential use of the UAVs, cooperative search operations with this kind of flying robots are not yet occurring. This research topic is a multidisciplinary area of study in its beginning. Several issues can be studied related to cooperative UAV for search operations, such as centralized versus decentralized control, path planning for cooperative flies, agent reasoning for UAV tactical planning, safety assessments, reliability in automatic target reconnaissance by cameras, agent coordination mechanisms applied for UAV cooperation and the application itself (e.g., patrolling, remote sensing, search and rescue operations supporting, etc.). This paper proposes a model that combines path planning algorithms, search patterns and multiagent coordination techniques to obtain a cooperative UAV model. Different path planning algorithms were studied aiming to get the most suitable to these kinds of operations, and the conclusions are presented. In addition, official documents of Search and Rescue operations are also studied in order to know the best practices already established for this kind of operations, and, finally, an overview of the coordination multi-agent theory is presented and evaluated to achieve the UAV coordination. The great goal for cooperative UAV is to achieve such performance that the performance of the group overcomes the sum of the individual performances isolatedly. Comparing two cooperative UAVs with two non-cooperative UAVs, simulations of previous work showed an average increase in efficiency by about $100 \%$. Besides, another aspect was simulated and evaluated: the impact of the density of the initial sweep. The computational simulations show a high potential of reducing even more the time of search. Furthermore, it is worth noting that the usefulness of cooperative UAVs is not only the increase in efficiency, but also the reduction of costs and risks for the crew.
\end{abstract}

Keywords: cooperative UAVs, autonomous UAVs, artificial intelligence, path planning algorithms, computational simulations. 


\section{INTRODUÇÃO}

Este trabalho propõe a utilização de mecanismos computacionais para modelar e avaliar o controle distribuído em Veículos Aéreos Não Tripulados (VANTs) cooperativos. O estudo de caso proposto é a utilização dos VANTs cooperativos em operações de busca num cenário de acidente em alto mar com espalhamento de destroços a partir de um ponto central. De acordo com o Comando da Aeronáutica do Brasil [2], esse é um dos cenários mais complexos em operações de busca e salvamento. Além do modelo de controle distribuído - que se originou de trabalhos anteriores [3] e teve a definição dos algoritmos computacionais aperfeiçoados -, simulações computacionais foram empregadas para auxiliar na avaliação dos principais parâmetros envolvidos no problema.

Dada a complexidade do cenário, somado ao fato de que as operações de busca constituem a fase mais importante numa operação de busca e salvamento [4], é bastante conveniente a utilização da computação na resolução desse problema. Principalmente porque existe uma grande quantidade de dados a serem tratados e de decisões a serem tomadas dinamicamente durante o processo de busca. Isso torna a coordenação pelo ser humano uma tarefa difícil.

Assim, a proposta deste trabalho é a utilização de mecanismos de coordenação da inteligência artificial, a escolha e a definição dos algoritmos de navegação mais apropriados à navegação de VANTs, e o emprego de simulações computacionais visando à parametrização da busca.

A seção 2 apresenta os VANTs: como surgiram, qual é o atual estado da arte, qual é a visão de futuro desses veículos e como chegar lá. A seção 3 apresenta a proposta computacional para a resolução do problema, que se fragmenta na análise dos principais algoritmos de navegação e na revisão dos mecanismos de coordenação provenientes da inteligência artificial. Por fim, a seção 4 e 5 apresentam, respectivamente, as simulações realizadas e as conclusões obtidas.

\section{VEÍCULOS AÉREOS NÃO TRIPULADOS}

O primeiro registro que se tem notícia de um VANT data de 1849, quando austríacos utilizaram balões interligados a cabos elétricos para lanças bombas em território inimigo. Pela definição atual, essa engenhoca já pode ser considerada um VANT. Nas duas Guerras Mundiais, diversos protótipos foram construídos e testes foram realizados. Porém, foram nas décadas de 70, 80 e 90 que os modelos de VANTs precursores dos que se conhece hoje foram desenvolvidos e aprimorados. Após essa evolução, verifica-se, desde o início do milênio, um crescimento bastante intenso do investimento e do interesse em tecnologias de veículos aéreos não tripulados. A previsão é que, dentro do setor aeronáutico, essa será a área de maior crescimento nesta década [17].

Ao longo da maior parte dessa evolução, os principais desafios se concentraram em aspectos mecânicos (construção da aeronave em si, aerodinâmica, etc). Nas últimas décadas, muito de trabalhou na tolerância a falhas dos sistemas envolvidos e na confiabilidade dos componentes eletromecânicos. Hoje, após décadas de avanço, os principais desafios estão 
relacionados ao controle do voo cooperativo dessas aeronaves, que é um problema predominantemente computacional.

Pesquisadores e relatórios governamentais apontam que, no futuro, múltiplos robôs voadores serão capazes de atuar, até sob a forma de enxames, cooperativamente e de modo autônomo [18], funcionando como uma rede coordenada de sensores que cumprirão missões complexas sem nenhuma intervenção humana [19]. Há acordo entre os pesquisadores da área que o principal desafio para obter cooperação em voo é a autonomia de decisão necessária a cada VANT. Conforme apontado pela Figura 1, ainda há um longo caminho para se chegar lá. Verifica-se que o VANT mais famoso em operação, o Global Hawk, ainda apresenta baixo grau de autonomia (entre os níveis 2 e 3). O UCAR Goal, indicado entre dos níveis 8 e 9 na figura, é um projeto que estava em vigor em 2005, mas as últimas notícias a respeito são que esse projeto foi congelado devido a restrições orçamentárias.

Cabe ressaltar que a autonomia de decisão, que se refere à capacidade do VANT tomar suas próprias decisões sem intervenção humana. Esta não deve ser confundida com a autonomia de voo, termo que também é bastante na pesquisa e desenvolvimento de aeronaves tripuladas e não tripuladas.

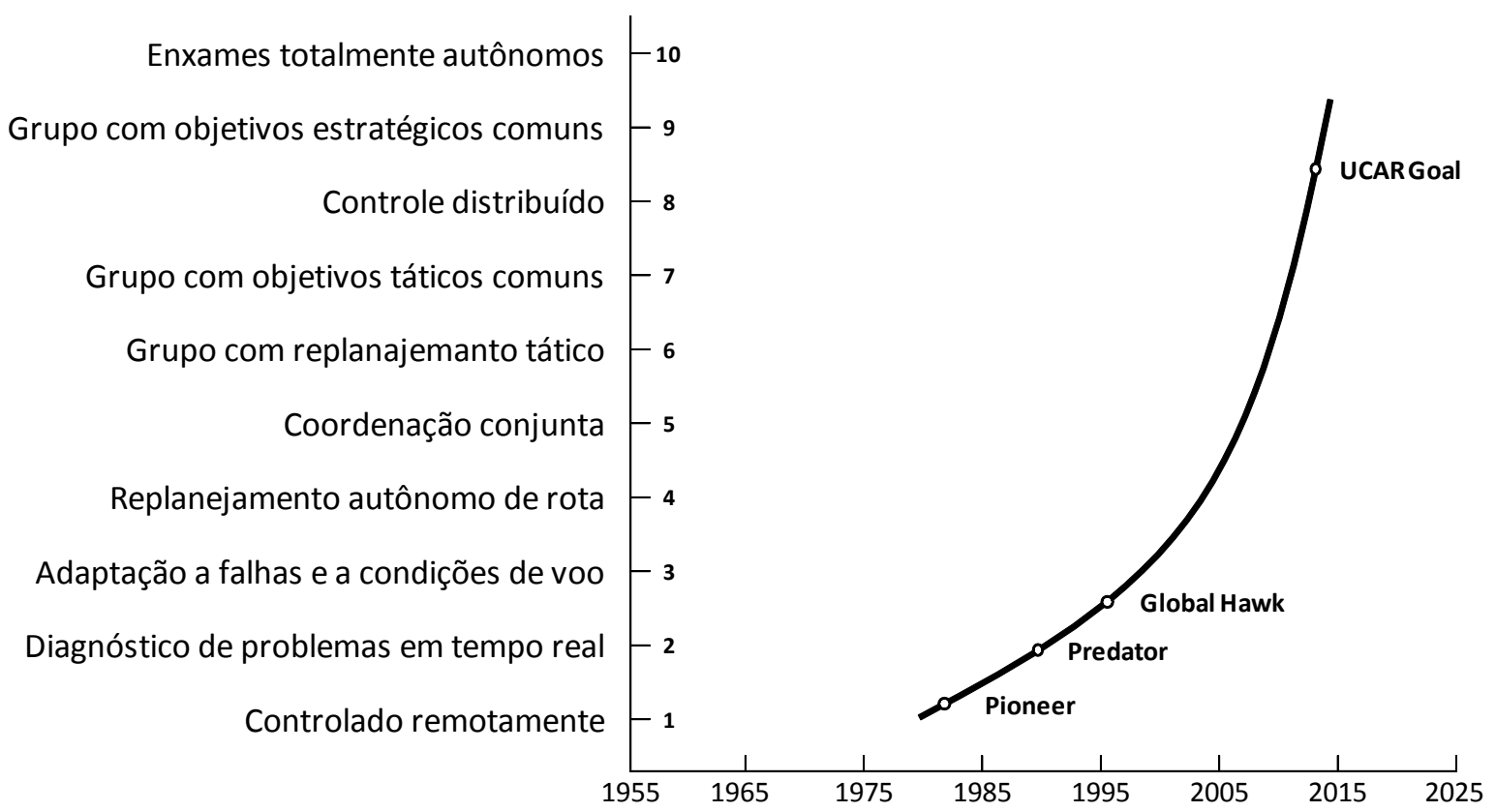

Figura 1. Níveis de autonomia de VANTs (adaptado de [5]).

Por fim, cabe ressaltar que a utilização de VANTs em operações de busca traz algumas vantagens em relação a operações de busca utilizando aeronaves tripuladas, como é feito atualmente. Esses robôs podem se submeter a uma exposição maior ao risco do que aeronaves tripuladas (voos em baixa altitude e, se necessário, até em condições climáticas severas, por exemplo), são capazes de executar operações de longa duração, sem necessidade de descanso (ao contrário dos voos tripulados) e apresentam menor consumo de combustível, podendo prolongar o tempo da busca sem reabastecimento.

Além disso, numa busca utilizando aeronaves tripuladas não é possível obter alto nível de cooperação, pois, além de toda a operação de busca e salvamento ser coordenada pelo Co- 
mandante de Operação (o que limita a quantidade de informações e o dinamismo envolvidos na coordenação), não se deve utilizar mais de uma aeronave tripulada para realizar buscas na mesma subárea. Essa situação geraria um estado de alerta prejudicial ao sucesso da operação, fazendo com que a tripulação tenha a sua atenção desviada das buscas para a navegação e a coordenação com outras aeronaves [8].

É nesse contexto, então, que se propõe utilizar técnicas da inteligência artificial de controle distribuído e algoritmos de navegação (como ferramentas de planejamento), bem como simulações computacionais para avaliação dos principais parâmetros envolvidos no processo de busca. A seção 3, a seguir, apresenta a modelagem computacional da proposta.

\section{ABORDAGEM COMPUTACIONAL}

Foi visto na seção 2 que o principal desafio para atingir o estágio de evolução esperado dos VANTs (os VANTs cooperativos) é a autonomia. É justamente a autonomia, a principal característica dos agentes inteligentes [1][9][14]. Por esse motivo, a teoria multiagente fornece uma poderosa base para obter a autonomia necessária para os VANTs cooperativos. Além disso, essa abordagem fornece um conjunto de mecanismos e protocolos de negociação, de coordenação e de tomada de decisão descentralizada em grupos de agentes cooperativos [13] - que é exatamente o que se pretende obter com os VANTs cooperativos.

Em relação os algoritmos de navegação, estes facilitam o planejamento de voo dos VANTs. Como a maior parte dos VANTs de pequeno porte navega pela programação da rota por meio de uma sequência de pontos (waypoints), este trabalho também avaliou, na subseção 3.2, os algoritmos de navegação mais adequados para gerar a sequência de pontos de navegação.

\subsection{Sistemas multiagentes}

Agentes inteligentes podem ser utilizados tanto em aplicações simples quanto, ao contrário do que possa parecer, em sistemas complexos e críticos [9] - que é o caso de um sistema distribuído de VANTs cooperativos. Dois exemplos de utilização de agentes inteligentes em sistemas complexos e críticos são os sistemas para exploração planetária CAMPOUT (Control Architecture for Multi-robot Planetary OUTpost) [7], desenvolvido pela NASA, e o MISUS (Multi-rover Integrated Science Understanding System) [6].

Desse modo, tendo segurança de que a teoria multiagente é um caminho promissor para obter o controle distribuído nos VANTs cooperativos, procuraram-se formas de obter a cooperação desses veículos. De acordo com [15], VANTs cooperativos trabalham em conjunto, levando em conta ações dos demais. Já [10] apontam que cooperar é coordenar com objetivo comum. E, por fim, [20] afirma que o maior problema na cooperação de agentes é a coordenação desses agentes.

A coordenação é o processo de garantir ações coerentes [12], visando à resolução de conflitos ou de interdependências de ações dos agentes [20] e aumentando o desempenho do grupo [1]. 
Sendo assim, foram mapeados da literatura os principais mecanismos de coordenação de agentes utilizados. São eles: (i) organização estrutural, cujo funcionamento se baseia na definição de papéis e responsabilidades dos agentes; (ii) acordos, cujo funcionamento se baseia na comunicação bilateral ou multilateral dos agentes visando à resolução de inconsistências e divisão de tarefas (um dos principais protocolos representantes dessa mecanismo de comunicação é o Contract Net [16]); (iii) compartilhamento de informações, em que a coordenação se baseia na troca de informações, aumentando, assim, a confiabilidade, a completeza e a precisão na tomada de decisão, reduzindo, também, o tempo de resposta; (iv) planejamento multiagente, que trata o problema de coordenação como um problema de planejamento, em que cada agente planejar previamente as ações para evitar inconsistências e conflitos com outros agentes; e, por fim, (v) negociação, em que um grupo de agentes busca consenso a respeito de alguma situação, podendo haver concessões.

Desses cinco mecanismos, o (i) e o (v) não são adequados ao problema que este trabalho deseja resolver. $\mathrm{Na}$ organização estrutural, nem sempre é possível prever com segurança as ações dos agentes. Na negociação, há perda de robustez, pois uma falha de comunicação ou falha de um agente pode, erroneamente, levar o sistema a não tomar uma decisão. Além disso, devido à necessidade de participação de todos os agentes, o tempo de processamento pode ser consideravelmente comprometido.

O mecanismo (ii) seria bastante adequado em operações envolvendo mais de dois VANTs, em que uma negociação mais sofisticada seja necessária para tomar alguma decisão. Como a simulação apresentada na seção 4 envolve apenas dois VANTs, não se utilizou esse mecanismo. Porém, trabalhos futuros a este irão utilizá-lo.

Assim, a simulação deste trabalho - que possui maior enfoque na combinação de técnicas computacionais de coordenação, simulação e de algoritmos - adotou os mecanismos (iii) e (iv). Cabe ressaltar que este trabalho parte de outro trabalho [3], acrescentando o uso intenso da simulação para obter outros resultados.

\subsection{Algoritmos de navegação}

A estratégia de busca empregada nas simulações foi baseada em [3]. Porém, aqui, foi dado maior enfoque na avaliação e definição dos algoritmos de navegação, e, na seção 4, no emprego de simulação para analisar os principais parâmetros envolvidos na operação de busca, visando, sempre, a redução do tempo de busca.

A busca se inicia com dois VANTs dividindo a área de busca entre si. Esses dois VANTs utilizam o padrão de busca "Rotas Paralelas", definido pelo Departamento de Controle do Espaço Aéreo (DECEA) [4] e ilustrado na Figura 2. Conforme pode ser observado na figura, esse padrão de busca não exige uma varredura com 100\% de densidade, sendo possível aumentar o espaçamento $(S)$ para obter uma varredura menos densa, porém com progressão mais rápida no espaço de busca. Na seção 4, esse parâmetro é objeto de simulação e análise.

Quando um dos VANTs detecta um objeto, este aciona o outro VANT para realizar uma varredura local (ao redor da primeira detecção). Ao ser acionado, o outro veículo aéreo se desloca até o local da detecção utilizando o algoritmo A*, cujo pseudocódigo se encontra na Figura 4. Esse algoritmo, que é o mais adequando à navegação de VANTs, gera uma sequência de pontos navegáveis de um ponto de origem até um ponto de destino. Na simulação 
apresentada na seção 4, a área de busca foi dividida em células, em que cada célula representa um nós navegável com conectividade 4, e considerou-se arestas de mesmo peso.

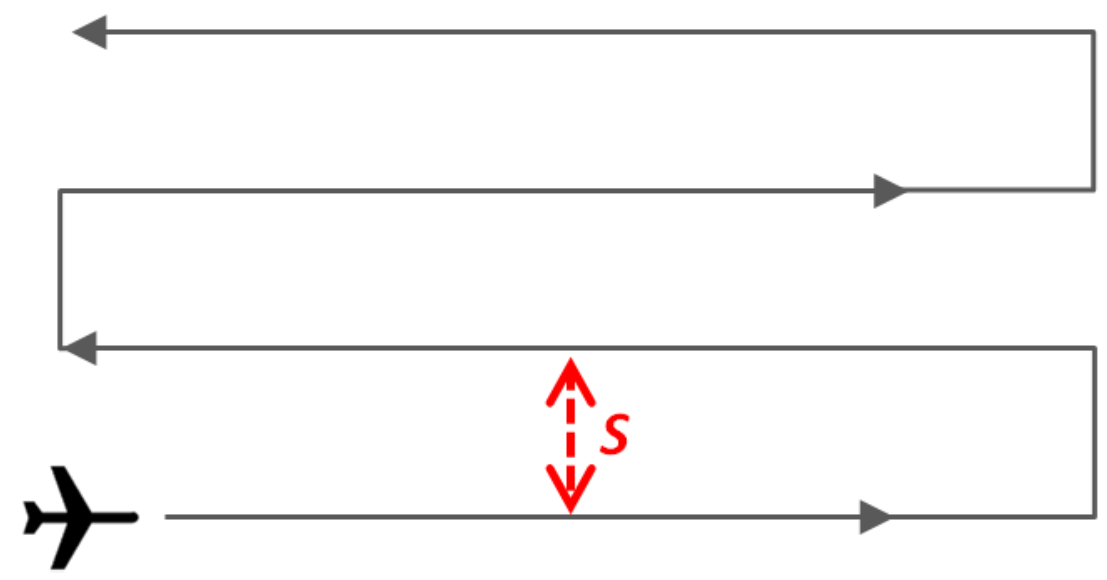

Figura 2. Padrão de busca Rotas Paralelas (Parallel Sweep). Adaptado de [4].

Outro algoritmo bastante famoso é o este algoritmo de Dijkstra, que é uma simplificação do algoritmo $\mathrm{A}^{*}$. A única diferença desse algoritmo com o $\mathrm{A}^{*}$ é que não é o custo estimado dos nós até o destino (função $H(n)$ da Figura 4) não é levado em consideração. Isso faz com que o algoritmo de Dijkstra explore uma área muito maior até encontrar o ponto de destino, fazendo com que seja mais lento que o $\mathrm{A}^{*} \mathrm{e}$, consequentemente, menos indicado para a utilização em VANTs. No entanto, o algoritmo de Dijkstra é mais indicado quando se deseja obter o caminho mínimo para visitar vários pontos, não importando a ordem [10].

A cada detecção de um objeto, cria-se uma área circular com 750 metros de raio ao redor da célula em que ocorreu a detecção. Essa área será varrida pelo VANT responsável pela busca local, que nada mais é do que o padrão de busca apresentado na Figura 2 com $100 \%$ de densidade de cobertura. Essa busca é iniciada assim que o VANT acionado chega nessa área.

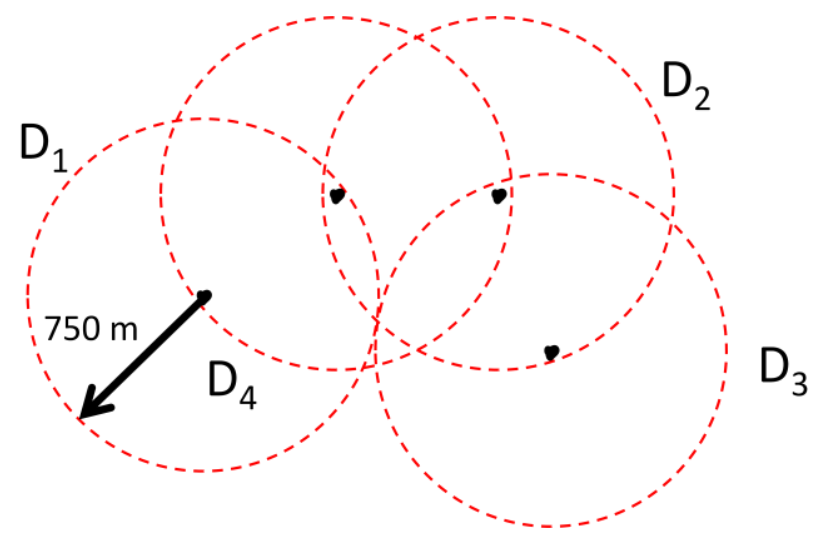

Figura 3. Exemplo de construções dinâmico do conhecimento do VANT com quatro detecções sucessivas $\left(\mathrm{D}_{1}, \mathrm{D}_{2}, \mathrm{D}_{3}\right.$ e $\left.\mathrm{D}_{4}\right)$.

Então, o VANT prossegue na busca local e a cada detecção a área alvo dessa varredura vai sendo formada dinamicamente com a incorporação de novas áreas circulares à original. Assim, a busca prossegue até varrer toda essa área (representada pela cor amarela na Figura 6, seção 4). Esse processo de formação dinâmica do conhecimento é ilustrado na Figura 3. 


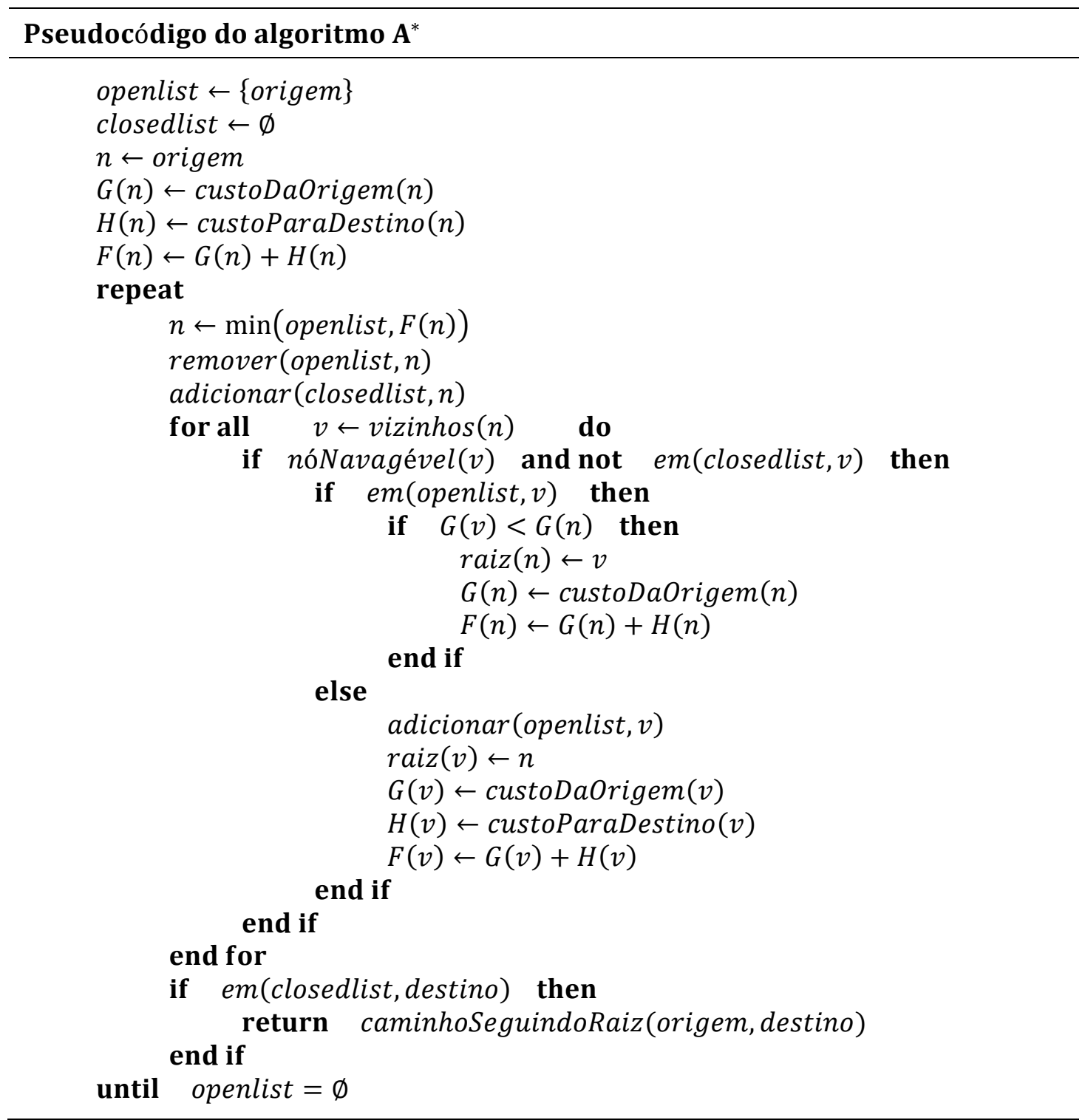

Figura 4. Pseudocódigo do algoritmo A*.

\section{SIMULAÇÃO}

Partindo dos resultados obtidos em [3], em que foi obtida uma redução média de 55\% no tempo de busca (empregando os mecanismos de coordenação multiagente, algoritmos de navegação e padrões de busca), as simulações deste trabalho tiveram como objetivo avaliar a influência a influência dos parâmetros nesse resultado para direcionar futuras melhorias.

Dessa forma, fixando um cenário representativo do caso médio, variou-se o espaçamento do padrão de busca "Rotas Paralelas" (parâmetro $S$ da Figura 2) - utilizado na fase inicial da busca e indicado quando se deseja uma cobertura rápida e uniforme. Quanto maior o espaçamento, mais rápida, porém menos densa, será a busca. No cenário utilizado nas simulações, havia vinte objetos espalhados por distribuição gaussiana com 500 metros de desvio padrão e mil (1.000) células de cem (100) metros de lado em uma área de busca quadrada com dez mil metros (10.000) de lado. 
Assim, ressalta-se que, com as simulações realizadas neste trabalho, não há qualquer pretensão de se esgotar o assunto e nem de sugerir diretrizes gerais que seriam adequadas a qualquer tipo de cenário. Trata-se de uma etapa anterior, porém necessária, às centenas de simulações com sorteio de cenários e análise estatística amostral, cujas observações, essas sim, resultarão em conclusões generalistas sobre a melhor parametrização da operação de busca utilizando VANTs cooperativos.

As simulações realizadas em [3] utilizaram somente espaçamento igual a uma célula $(\mathrm{S}=1)$, ou seja, o padrão "Rotas Paralelas" realizava a varredura com $100 \%$ de cobertura. Conforme ilustrado na Figura 5, o aumento do espaçamento tende a reduzir o tempo da busca. Mas não necessariamente essa redução do tempo de busca traz benefícios, pois a probabilidade de detecção de objetos também é reduzida. Observa-se que, em certas situações, podem-se detectar dezenove (19) de vinte (20) objetos e até mesmo nenhum objeto. Essas situações, indicadas pelo rótulo em vermelho da figura 4 , ocorreram para $\mathrm{S}$ igual a 2,4 e 7 , respectivamente.

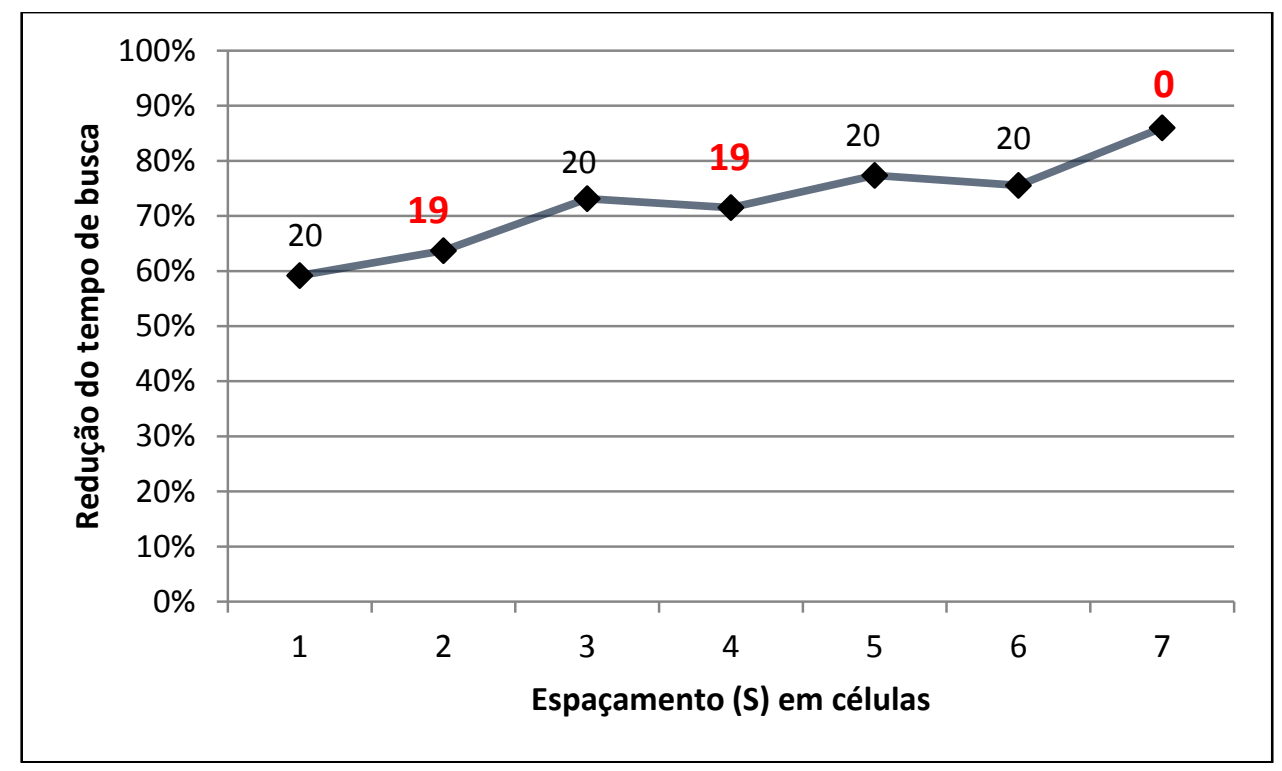

Figura 5. Variação do espaçamento $(S)$ do padrão de busca "Rotas Paralelas". S = 1 significa $100 \%$ de cobertura; $\mathrm{S}=2$ significa que a distância entre as "idas e vindas" do VANT no padrão "Rotas Paralelas" é de duas células (200 metros); $\mathrm{S}=3$, três células; e assim por diante. Os rótulos na linha do gráfico indicam o número de objetos detectados de um total de vinte (20) objetos perdidos.

Durante as simulações, observou-se que outros parâmetros também podem influenciar bastante na obtenção do espaçamento ótimo do padrão "Rotas Paralelas": (i) o número de objetos espalhados; (ii) o desvio padrão do espalhamento gaussiano dos objetos; (iii) e as parametrizações da algoritmo de busca local.

O número de objetos espalhados pode variar de acordo com o impacto e o tamanho do acidente, já o desvio padrão do espalhamento gaussiano, de acordo com o [4], pode variar de acordo com a deriva dos objetos em alto mar. Esta, por sua vez, varia de acordo com o tempo decorrido desde o acidente, a velocidade do vento e a corrente marítima. Quanto maior o espalhamento dos objetos e menor o número de objetos, menor tende a ser a probabilidade de 
detecção. Sendo assim, uma forma de aumentar essa probabilidade de detecção é reduzir o espaçamento $(S)$ da busca inicial pelo padrão de busca "Rotas Paralelas", tornando, consequentemente, a varredura inicial mais densa.

Em relação ao algoritmo de busca local, este pode sofrer bastante influência do raio de atualização do conhecimento (estabelecido em 750 metros nas simulações), que deve estar adequado ao desvio padrão do espalhamento gaussiano. O raio de atualização deve ser o menor possível, mas garantindo que a detecção de todos os objetos seja possível. Num cenário em que há poucos objetos com grande espalhamento, por exemplo, esse raio de atualização do conhecimento precisa ser maior para que, a cada detecção, a área de busca local se estenda alcançando os outros objetos (conforme ilustrado na Figura 3).

A Figura 6 ilustra o instante final da busca para cada parâmetro $S$ utilizado. Na simulação, a busca foi dada por encerrada quando todos os objetos foram encontrados ou quando o VANT responsável pela busca local varreu toda a área com maior probabilidade de detecção, representada pela área amarela na Figura 6.

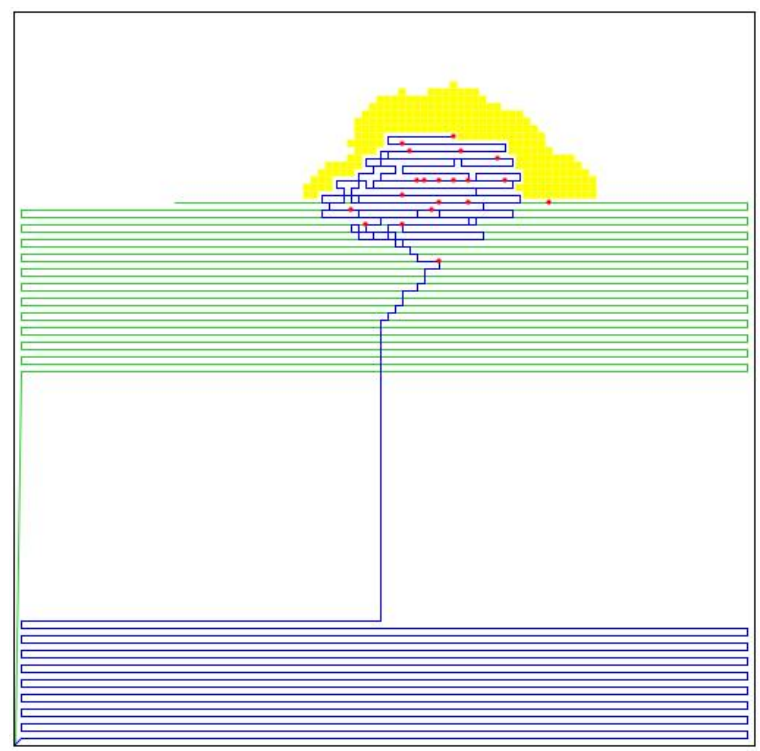

(a) $\mathrm{S}=1$.

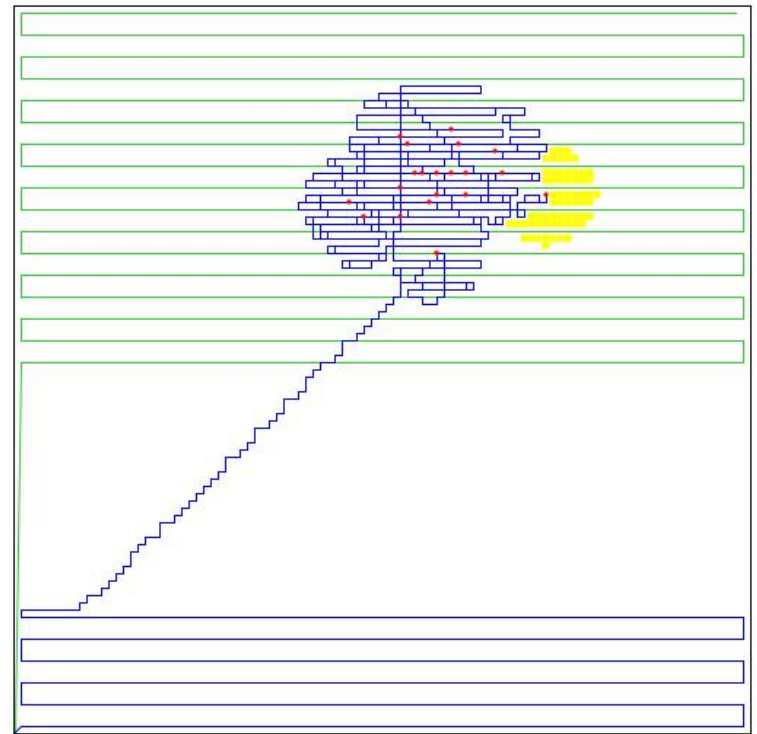

(c) $\mathrm{S}=3$.

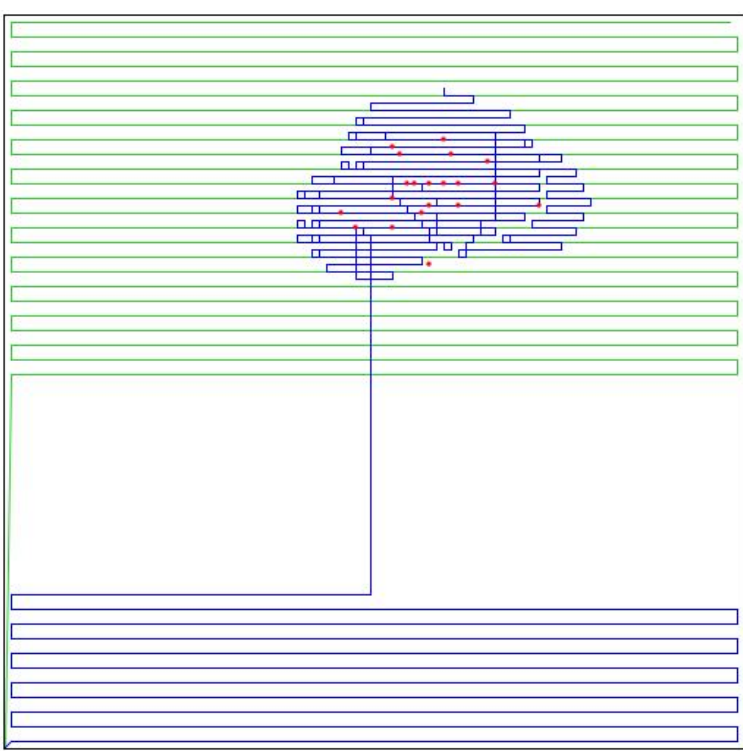

(b) $\mathrm{S}=2$. Apenas 19 objetos detectados.

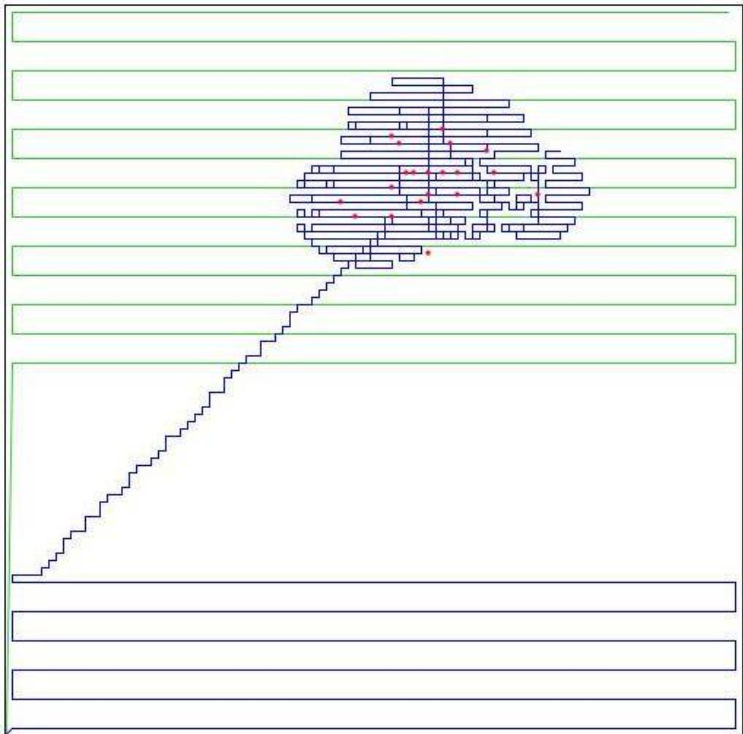

(d) $\mathrm{S}=4$. Apenas 19 objetos detectados. 


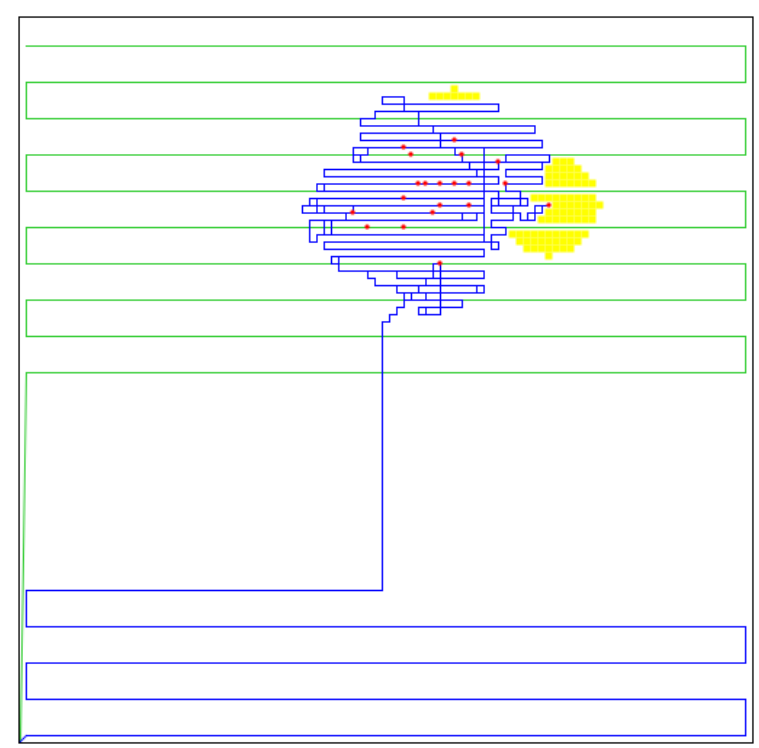

(e) $\mathrm{S}=5$.

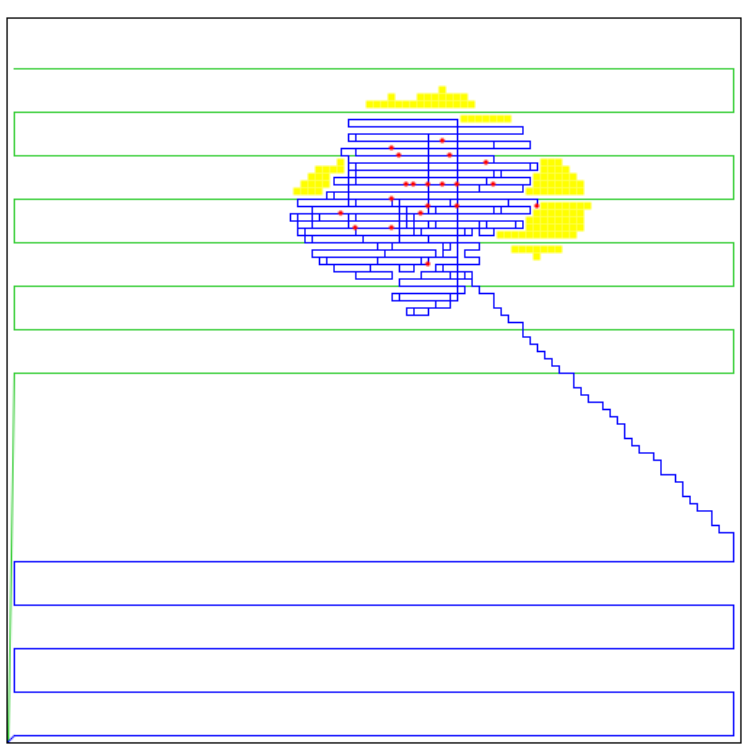

(f) $S=6$.

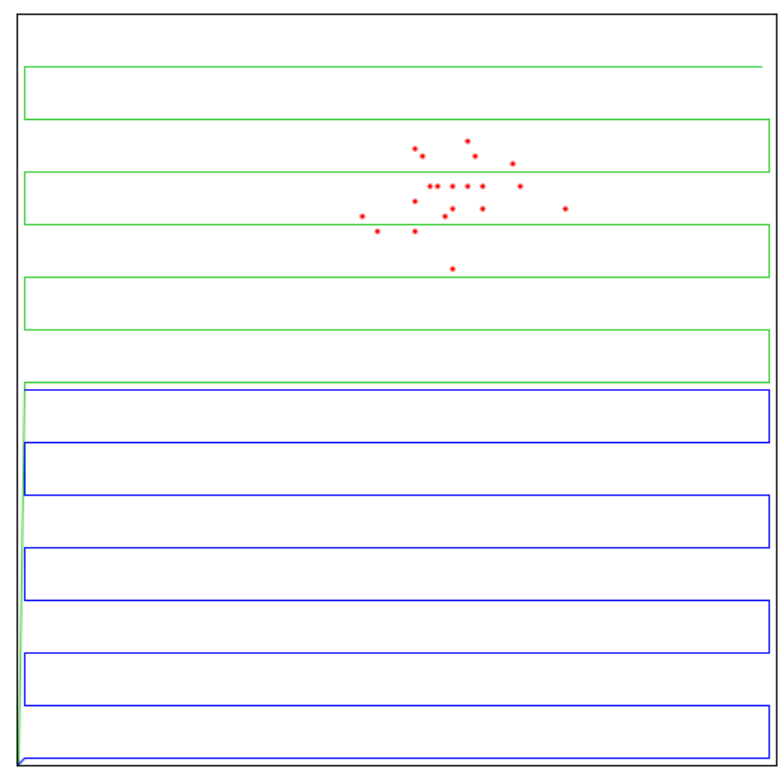

(g) $\mathrm{S}=7$. Nenhum objeto detectado.

Figura 6. Registro da navegação dos VANTs variando o espaçamento $(S)$ do padrão de busca "Rotas Paralelas". A área amarela nas figuras (a), (c), (e) e (f) representam a área de maior probabilidade (armazenada na forma de conhecimento do agente - VANT), cuja construção é feita dinamicamente.

Observa-se que nas Figuras 6(a), 6(c), 6(e) e 6(f), o VANT responsável pela busca local encontrou todos os objetos perdidos antes de varrer a região de maior probabilidade (representada pela área amarela e construída dinamicamente por meio das sucessivas detecções).

Nas Figuras 6(b) e 6(d), mesmo varrendo toda a região de maior probabilidade de conter objetos (toda a região amarela foi "consumida"), o VANT responsável pela busca local encontrou apenas 19 objetos, de um total de 20 . Isso ocorre porque havia um objeto mais afastado do foco de espalhamento - que não foi detectado pela varredura inicial empregando o 
padrão "Rotas Paralelas" - e todos os outros objetos detectados estão a mais de 750 metros de distância desse objeto mais afastado - distância mínima necessária para que seja incorporado à área de maior probabilidade (Figura 3).

\section{CONCLUSÃO}

Este trabalho partiu de resultados obtidos em [3] e, visando melhorar esses resultados, realizou simulações com o objetivo de avaliar a influência dos parâmetros envolvidos numa operações de busca.

Os resultados mostram que, empregando técnicas de inteligência artificial e algoritmos de navegação, a utilização de VANTs cooperativos em operações de busca pode reduzir consideravelmente o tempo de busca em uma operação de busca e salvamento. Com isso, é possível aumentar a chance de sobrevivência de vítimas não fatais. Além disso, os VANTs possuem certas vantagens inerentes a sua natureza: possibilidade de maior exposição ao risco do que uma aeronave tripulada; possibilidade de compartilhamento de uma mesma subárea; e capacidade de realizar operações de longa duração sem descanso.

Pelas simulações apresentadas na seção 4, observa-se um potencial de redução ainda maior pelo ajuste do espaçamento $(S)$ do padrão de busca "Rotas Paralelas" - parâmetro que não deve ser negligenciado numa situação de busca real. No entanto, não existe um ajuste perfeito do espaçamento ótimo para qualquer cenário. $\mathrm{O}$ aumento do espaçamento implica a redução da probabilidade de detecção dos objetos (podendo até resultar na detecção de nenhum objeto). Além disso, características do cenário, como, por exemplo, o espalhamento e o número dos objetos perdidos, possuem influência no ajuste do espaçamento $(S)$.

Com o intuito de tirar conclusões generalistas, outras simulações estão sendo realizadas utilizando o sorteio de cenários e avaliação estatística amostral.

Por fim, outro caminho a seguir, poderia ser a criação de uma heurística que, para cada cenário de busca, calculasse os parâmetros da busca a partir do número de objetos e do desvio padrão do espalhamento. Como esse cálculo seria feito apenas uma vez, antes de iniciar as buscas, é totalmente viável que seja utilizado numa operação real.

\section{Agradecimentos}

Os autores agradecem ao CNPq (Conselho Nacional de Desenvolvimento Científico e Tecnológico) pelo apoio concedido na forma de bolsa de mestrado.

\section{REFERÊNCIAS}

[1] Bellifemine, F., Caire, G., and Greenwood, D. (2007). Developing multi-agent system with JADE. Wiley.

[2] Centro de Comunicação Social da Marinha. Término das buscas do voo 447 da Air France. Força Aérea Brasileira, 2009. Disponivel em: 
$\langle$ http://www.fab.mil.br/portal/capa/index.php?mostra=3311 >. Acesso em: 2011 Julho 12 .

[3] Chaves, A. N. and Cugnasca, P. S. (2012). Cooperative UAVs using multi-agent coordination techniques for search operations. In Anais do VI Workshop-Escola de Sistemas de Agentes, seus Ambientes e apliCações (WESAAC 2012), Florianópolis, SC, Brasil. No Prelo.

[4] DECEA (2009). Manual de busca e salvamento. Relatório Técnico, Comando da Aeronáutica.

[5] DoD (2005). Unmanned aircraft systems roadmap 2005-2030. Relatório Técnico, Department of Defense - DoD.

[6] Estlin, T., Gaines, D., Fisher, F., and Castano, R. (2005). Coordinating multiple rovers with interdependent science objectives. In Proceedings of the fourth international joint conference on Autonomous agents and multiagent systems, AAMAS '05, pages 879886, New York, NY, USA. ACM.

[7] Huntsberger, T., Pirjanian, P., Trebi-ollennu, A., Nayar, H. D., Ganino, A. J., Garrett, M., Member, A., Joshi, S. S., and Schenker, P. S. (2003). Campout: A control architecture for tightly coupled coordination of multi-robot systems for planetary surface exploration. IEEE Trans. Systems, Man \& Cybernetics, Part A: Systems and Humans, $33: 550-559$.

[8] IMO/ICAO (2003). International aeronautical and maritime search and rescue manual. Technical report, International Maritime Organization and International Civil Aviation Organization, London, United Kingdom.

[9] Jennings, N. R. and Wooldridge, M. (1998). Applications of intelligent agents, pages $3-$ 28. Springer-Verlag New York, Inc., Secaucus, NJ, USA.

[10] Lester, P. A* path finding for beginners. PolicyAlmanac.org, 2005. Disponivel em: <http://www.policyalmanac.org/games/aStarTutorial.htm>. Acesso em: 14 de dezembro 2011.

[11] Luck, M., McBurney, P., Shehory, O., and Willmott, S. (2005). Agent Technology: Computing as Interaction (A Roadmap for Agent Based Computing). AgentLink.

[12] Nwana, H., Lee, L., and Jennings, N. (1996). Coordination in software agent systems. British Telecom Technical Journal, 14 (4):79-88.

[13] Pechoucek, M. and Sislak, D. (2009). Agent-based approach to free-flight planning, control, and simulation. IEEE Intelligent Systems, 24(1):14-17.

[14] Russell, S. and Nowig, P. (2009). Artificial Intelligence: A Modern Approach. Prentice 
Hall, 3rd. edition.

[15] Shima, T. and Rasmussen, S. (2008). UAV Cooperative Decision and Control: Challenges and Practical Approaches. Society for Industrial and Applied Mathematics, Philadelphia, PA, USA.

[16] Smith, R. (1980). The contract net protocol: High-level communication and control in a distributed problem solver. IEEE Transactions on Computers, C-29(12):1104-1113.

[17] Teal Group (2011). World unmanned aerial vehicle systems - 2011 market profile and forecast. Technical report, Teal Group Corporations, Fairfax, VA, EUA.

[18] UAS Center of Excellence (2010). U.s. army roadmap for unmanned aircraft systems 2010-2035. Technical report, Fort Rucker, Alabama.

[19] Vachtsevanos, G., Tang, L., and Reimann, J. (2004). An intelligent approach to coordinated control of multiple unmanned aerial vehicles. In American Helicopter Society International, I., editor, In Presented at the American Helicopter Society $60^{\text {th }}$ Annual Forum, Baltimore, MD.

[20] Wooldridge, M. (2009). An Introduction to MultiAgent Systems. John Wiley \& Sons, 2nd. ed. 